\title{
Pharmaceutical supply chains in Africa - overcoming unique transport security challenges
}

\author{
Bryan Goshorn • Craig Usswald
}

Received: 6 August 2014 / Accepted: 20 August 2014 / Published online: 26 October 2014

(C) Springer Science+Business Media New York 2014

\section{Promoting vigilance in Africa's complex and hyper-dynamic environment}

According to the World Health Organization, Sub-Saharan Africa still has the worst health conditions of anywhere in the world. Stated in their World Health Statistics 2014, a major shift has occurred elsewhere around the globe, where fewer and fewer deaths are caused by infectious diseases. In many African nations, however, $70 \%$ of premature deaths are still caused by infectious diseases and related conditions, and the life expectancy for both men and women is still less than 55 years. Diseases like Ebola, malaria, cholera, tuberculosis and AIDS, as well as violence, widespread poverty and drought have strained an already inadequate and overburdened healthcare delivery system to the breaking point. As a result, a significant number of poor and disadvantaged Africans are not getting the medicine they need. While pharmaceutical distribution is not the only sector impacted by these challenges, the consequence of these late or missing deliveries can impact lives.

Part of this is a function of constraints caused by internal and external politics, economics, climate, religion and infrastructure that will need concerted global effort and investment and many years to address. But much of it can be improved simply by determining that the right medicines and medical supplies reach the right people in the right ways. Much of it is a matter of basic transport and supply chain management.

Africa is experiencing the highest freight movement volumes ever seen on the continent. The supply of resources has not kept pace with the ever-changing demand, and much of the freight movement efficiency is hampered by inadequate planning. When outside organizations design transport security plans on the African continent, they tend to leverage the same global leading practices that have worked so well in other contexts. But, because Africa is such a unique continent demographically, medically, and economically, employing these same practices in Africa without significant modification can lead to regrettable and substantial gaps in execution. These execution gaps can be minimized by intimately knowing and adjusting to the African environment.

B. Goshorn $(\bowtie) \cdot$ C. Usswald

Deloitte Consulting LLP, Strategy and Operations, Washington, D.C., USA

e-mail: brgoshorn@deloitte.com 
For example, it is difficult to design an effective medical supply chain in Africa without understanding that pharmaceutical shipments represent high value goods. This makes their supply chains more risk-prone than in other places, and is why it is crucial that the precautions taken to secure transport are not compromised. Special care must be taken to counter the high risk of external threats like hijacking, load diversion, pilfering and occasionally even terrorism, by closing the gaps that are evident in standard security systems.

\section{Challenges and solutions}

Several factors complicate transport security in these environments. While global leading practices can be used to build the security strategy foundation, specific tailoring is essential to be relevant in these resource-constrained areas. In order to close any remaining gaps, additional enhancements should be incorporated into the security strategy to minimize the risk to both cargo and drivers. Creating strict accountability and visibility within each phase of transport from origin to destination is crucial for effectiveness. This article explores examples of tailored global leading practices and the recommended security enhancements designed to maintain load integrity for both transporter and shipper.

\section{Pickup}

Global leading practices

- Fill fuel tanks prior to arrival: Arriving with full fuel tanks allows the driver to maximize the distance between pickup location and refueling, thereby reducing probability of effective tracking and monitoring by potential thieves. The geographies near pickup and delivery are the most prone to theft and overall risk; having a full fuel tank upon arrival will enable drivers to spend less time stationary and more time in motion.

- Minimize "time in yard" of loaded and dropped trailers: If loaded and dropped trailers are a necessity, it is crucial to minimize the time that these trailers are idle in the yard in order to reduce vulnerability for potential theft.

- Involve the driver during loading: A driver who is present and actively viewing the loading process has the potential to discourage "inside job" and theft collusion activity from shipping staff.

- Administer security seals on trailer/container with driver present, but not by the driver: Broken seals are the first indication of a compromised load and new seals should be placed on the trailer/container only by shipper staff to maximize transport security continuity. Seal colors and types should not be correlated to load value as the probability of thieves visually identifying and selectively targeting high value loads may increase.

Enhanced practices

- Mandate security awareness training: Prior to driver arrival, security awareness training should be mandated for drivers/staff to establish intimate cognizance of 
common enterprise security and geographic risks. In many cases in Africa, significant regions of heightened armed banditry and terrorist activity exist. In these areas, preparations for bartering/payment (e.g., food, water, money) should be planned for in preparation for entrance into extremely dangerous delivery sectors. With this in mind, it is highly encouraged to develop and maintain a risk matrix and to plan accordingly when making deliveries to these high risk locations.

- Practice excessive vigilance with weather forecasting and monitoring: Mud, floods, or other environmental conditions can stifle vehicle movement during travel. In extremely dangerous delivery locations, environmental conditions can expose both driver and cargo to undesirable situations. Improper monitoring of weather conditions may not only increase the risk of cargo theft, but also presents life or death situations for drivers. Therefore, these risks should be identified and monitored as a prerequisite prior to shipment departure.

- Paint trailers unusual colors: Minimize trailer branding and alternate color schemes (if able) to delivery locations. Although it is common for trailers to have registration numbers painted on top in large black font for airborne surveillance, plain white trailer coloring presents challenges for authorities on the ground when attempting to track and/or recover. (Osterberg)

\section{In-Transit}

Global leading practices

- Exert maximum vigilance during facility departure and arrival: Thieves perform substantial surveillance on roads and gateways to/from facilities. Heightened monitoring during these phases of shipment is critical.

- Assign team drivers to high value loads: Additional manpower can minimize the amount of stops required, while also providing an extra set of eyes to monitor cargo when stops are required. (Osterberg)

- Develop a relationship with local law enforcement: Developing a relationship with local law enforcement can provide drivers with an added layer of situational awareness by communicating and taking direction to circumvent potentially dangerous locations.

- Leverage technology and monitor vehicles/loads throughout the journey: Utilize robust transport security monitoring programs including round the clock customer service to monitor vehicles and cargo (e.g., geofencing, tracking devices, electronic seals) and communicate with drivers throughout all segments of the journey.

\section{Enhanced practices}

- Minimize in transit vehicle branding: Minimize vehicle branding when carrying high value goods, especially when traveling into dangerous delivery sectors. Even when trailer branding is minimized or even eliminated, regulations commonly require company identification on tractor/cab front doors. When in-motion in extremely high risk areas, it is encouraged (if locally permissible) to cover or "mask" any identifying features using any means necessary (e.g., cardboard, paper covering). 
- Exert maximum vigilance during forced and/or deceptive stops: When approaching roadblocks or local police checkpoints, be extremely vigilant as cargo crime gangs are extremely prevalent and frequently pose as local authorities. (International Road Transport, 2005)

- Pursue in-field, second tier Proof of Delivery (POD) signatures to establish chain of custody: It is sometimes essential to cross-dock in the field when "brick and mortar" facilities are not present. Field cross docking is common in remote locations requiring specialized transport (e.g., 4x4, ATV, camel, ferry) and requires an additional layer of accountability such as photographs of recipients for real-time authorization confirmation because security mechanisms are rarely present. It is not recommended to allow transfer of goods without maximum traceability and accountability.

\section{Delivery}

Global leading practices

- Avoid waiting times outside of normal working hours: Forecasts arrival times should be planned within normal business operating hours and also in coordination with facility receiving staff.

- Perform a comprehensive verification of security devices, items and associated paperwork upon arrival: Verify security hasn't been compromised, paperwork and order remains correct, status of temperature recording devices are within tolerance, and also that the transport and storage conditions have been maintained throughout the journey.

- Receiving staff should be properly badged and in official uniforms: Even once inside the delivery point security perimeter, remain vigilant by confirming that individuals who are unloading the vehicle are properly badged and valid employees.

\section{Enhanced Practices}

- Develop performance management structures to reward, penalize, and enforce the seal strategy: Formal debrief sessions with each driver upon completion are imperative. These sessions should include examining loads to confirm each "cut" seal matches 1:1 to each POD. The driver's ability to carry the next load should be contingent on successful completion of the existing loads debrief.

- Perform random audits and spot checks to reduce probability of potential driver/receiver collusion. Collusion, commonly present at some degree, can be reduced by maximizing security presence and fortification with structured processes and procedures. Requiring drivers to present ID cards and sign with "block lettering" fields on POD documentation assists with this reduction in risk. Limiting contact and movement of parties at facilities (e.g., hole in the wall at secure distribution facility) also helps to minimize instances of collusion.

- Establish robust, pre-arranged processes for returned goods: The risk of exploitation during returns is highly due to the fact that the majority of security focuses on outbound finished goods. As a result, processes should be in place for 
return activities. Ad hoc pickups are highly discouraged and proper paper trails, procedures, and processes should be designed and adhered to. It is highly recommended to confirm that the driver has an official copy of a memo from the CEO of the transport company prohibiting pickup of returns without pre-authorization. When dealing with high-valued pharmaceuticals especially, compliance is paramount.

\title{
Establishing long term effectiveness
}

If organizations plan to operate pharmaceutical-based supply chains in the African landscape, robust and compliant transportation security strategies are imperative. In addition to ensuring ISO (Kaye) 28000 compliance, organizations should promote enterprise planning, training, rigid execution, and robust audit procedures for continuity of shipment and the safety of crew and equipment.

Furthermore, it's important to keep in mind that Africa has certain unique issues impacting its pharmaceutical supply chain. Therefore, to successfully develop an efficient, streamlined supply chain, potential transport risks must be anticipated at the start of the process and a plan of action must be developed to reduce the probability of these risks occurring. A supply chain approach which successfully encompasses demonstrated supply chain principles and processes combined with the customization necessary to address Africa's distinctive circumstances can not only enable successful and profitable delivery of medicine to the Africans who need it, but will help save the lives of the African population.

\section{References}

International Road Transport, IRU Road Transport Security Guidelines, 2005

Kaye, Simon, 10 innovations for Cargo and Supply Chain Security, Journal of Commerce, (2011)

Osterberg, Don, Security Whitepaper: Keeping your Cargo Safe, Schneider National, (2010)

World Health Organization, World Health Statistics 2014

\begin{abstract}
About Deloitte
Deloitte refers to one or more of Deloitte Touche Tohmatsu Limited, a UK private company limited by guarantee, and its network of member firms, each of which is a legally separate and independent entity. Please see www.deloitte.com/about for a detailed description of the legal structure of Deloitte Touche Tohmatsu Limited and its member firms. Please see www.deloitte.com/us/about for a detailed description of the legal structure of Deloitte LLP and its subsidiaries. Certain services may not be available to attest clients under the rules and regulations of public accounting.

This publication contains general information only and Deloitte is not, by means of this publication, rendering accounting, business, financial, investment, legal, tax, or other professional advice or services. This publication is not a substitute for such professional advice or services, nor should it be used as a basis for any decision or action that may affect your business. Before making any decision or taking any action that may affect your business, you should consult a qualified professional advisor.

Deloitte shall not be responsible for any loss sustained by any person who relies on this publication.
\end{abstract}

